\title{
SUPPORTING FIRST RESPONDERS BY TERRESTRIAL BLUETOOTH-BASED TRAFFIC MONITORING IN THE CASE OF LARGE-SCALE EVENTS
}

\author{
GABY GURCZIK, RONALD NIPPOLD \& FREDERIK NIMPHIUS \\ German Aerospace Center, Germany
}

\begin{abstract}
Within the VABENE++ project (2014-2018) of the German Aerospace Center (DLR), powerful softand hardware tools are being developed to aid public authorities and organisations with security responsibilities as well as traffic authorities when dealing with risk and disaster management in the case of large scale events. The goal is to manage the required rescue logistics and the nearby traffic flow even under extreme conditions and, thus, enabling response teams to rapidly reach the locations where they are needed. To efficiently react in the case of large scale events, professional responders highly depend on a quick and reliable overview of the current situation. This includes, in particular, the estimation of affected areas and herein the number of people who need to be evacuated, as well as the assessment of the current traffic situation along the arrival and departure roads. In the present paper we describe the application of a terrestrial Bluetooth-based monitoring system for the purpose of traffic and person monitoring during large scale events. Using the example of a worldwide renowned open air festival, a case study was carried out that accompanied the actual operating business of the event management. The aim of this case study was, on the one hand, to automatically collect anonymised data of the current traffic situation along the arrival roads and, on the other hand, to gain data of the local number of persons at specific points of interest in the event area throughout the entire duration of the festival. The results of the case study indicate that the usage of locally deployed Bluetooth-based sensors allows adequate continuous time measurement enabling the registration of reliable situation changes in the local concentration of people over the course of the day.
\end{abstract}

Keywords: large scale events, disaster management, traffic monitoring, Bluetooth.

\section{INTRODUCTION}

The VABENE++ project of the German Aerospace Center (DLR) aims to develop powerful tools in order to aid public authorities and organisations with security responsibilities as well as traffic authorities when dealing with disasters and large public events. The goal is to efficiently manage the required rescue logistics and the nearby traffic flow even under extreme conditions, thereby enabling response teams to rapidly reach the locations where they are needed. Research in the VABENE++ project focuses on areas such as simulation and large-scale traffic modelling, aerial and terrestrial traffic monitoring, traffic risk assessment, data fusion, data management, and further developing Web technologies in a GIS environment. For this purpose, data from several platforms and with different spatialtemporal references such as high resolution satellite images, real-time aerial images and terrestrial sensors like Floating Car Data (FCD) and Bluetooth-based sensors are used. By combining the different perspectives of the sensor systems, valuable information for decision support can be derived.

To efficiently react in case of large scale events, professional responders highly depend on a quick und reliable overview of the current situation. This includes in particular the estimation of affected areas and herein the number of people who are in need to be evacuated, as well as the assessment of the current traffic situation along the arrival and departure roads.

In the present paper we describe the application of terrestrial Bluetooth-based sensors for the purpose of traffic and person monitoring during large scale events. By the example of a 
worldwide renowned open air festival, a case study was carried out that accompanied the actual operating business of the event. The aim of this case study was, on the one hand, to automatically collect anonymised data of the current traffic situation along the arrival roads, and on the other hand, to gain data of the number of persons at specific local points of interest on the event area throughout the entire duration of the festival.

Within the paper, we first explain the technique used for the monitoring process. Afterwards, we present the design and implementation of the case study followed by the results and their evaluation.

\section{BLUETOOTH MONITORING METHOD}

Bluetooth and WiFi devices are available in a number of vehicles (e.g. in terms of mobile devices such as smartphones and headsets as well as in-vehicle systems like satnav or car radio) and thus, allow the detection of motions of persons and goods [1], [8]. Detectable traffic objects are vehicles, pedestrians, cyclists as well as passengers of public transport carrying Bluetooth/WiFi equipped devices. Since every Bluetooth- or WiFi-enabled device is uniquely identifiable due to its electronical device address (so called Media Access Control address (MAC)), the monitoring itself is based on the detection of anonymised MAC addresses [2]. For this purpose, the MAC addresses are automatically linked to a timestamp consisting of date and time and the geographical coordinates of the stationary sensor. The Anonymisation is based on a rotary Salted-Hash procedure (i.e. coding is done via a periodically changing code that uses random values) and thus, ensures proper privacy handling of the traffic participant's data. Depending on the used hardware (Bluetooth/WiFi adapter), the detection radius ranges (Fig. 1) from 1-2 meters for class 3 Bluetooth adapters, to 25 meters for class 2 and around 100 meters for class 1 adapters, whereby the detection range can heavily depend on environmental impacts, e.g. in case of multipath scattering, or due to atmospheric effects.

The DLR Institute of Transportation Systems developed a prototypical sensor system that enables the monitoring of traffic objects via radio-based technologies such as Bluetooth and WiFi. Therefore, the sensor system (Fig. 2(c)) consists of several parts: A main sensor module (shown in dark blue) consists of radio interfaces supporting Wi-Fi, Bluetooth, GPS and GSM/UMTS, as well as a computer unit (brown box). A waterproof casing (dark gray)

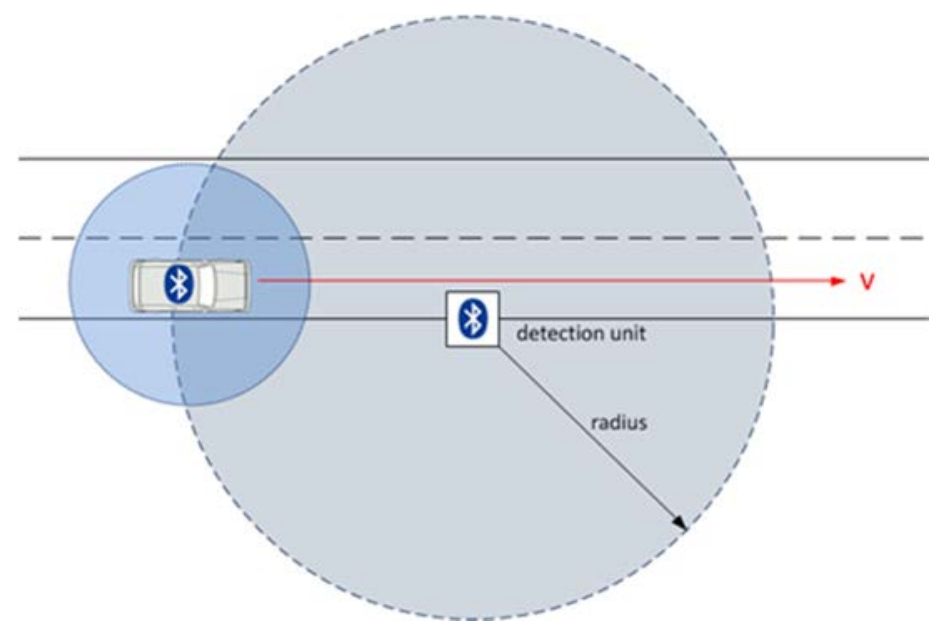

Figure 1: Functional principle of Bluetooth-based traffic monitoring. (Source: DLR.) 


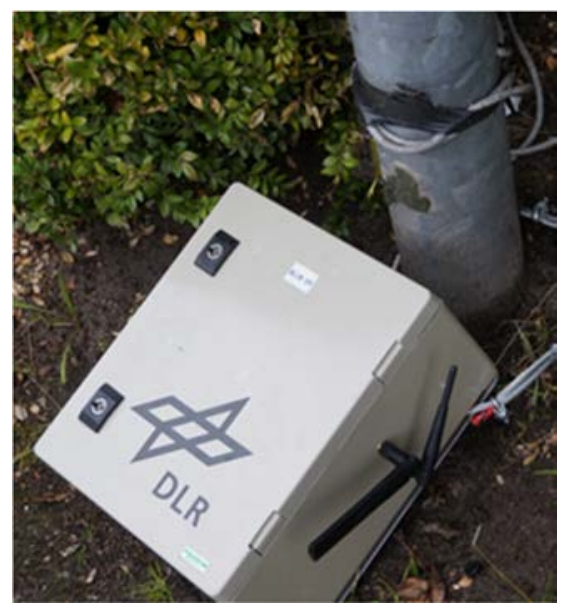

(a)

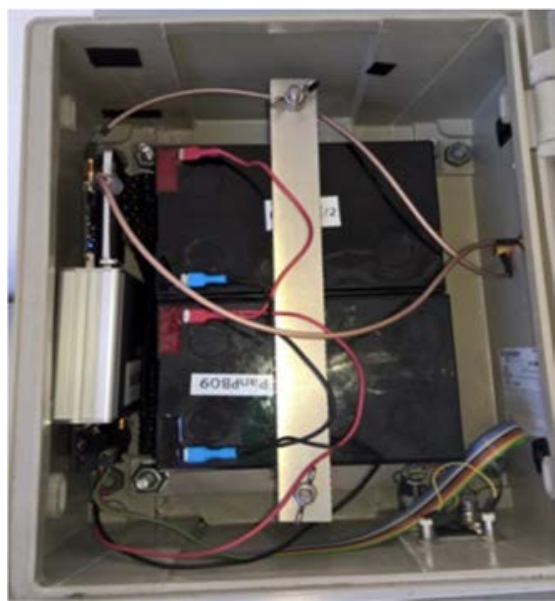

(b)

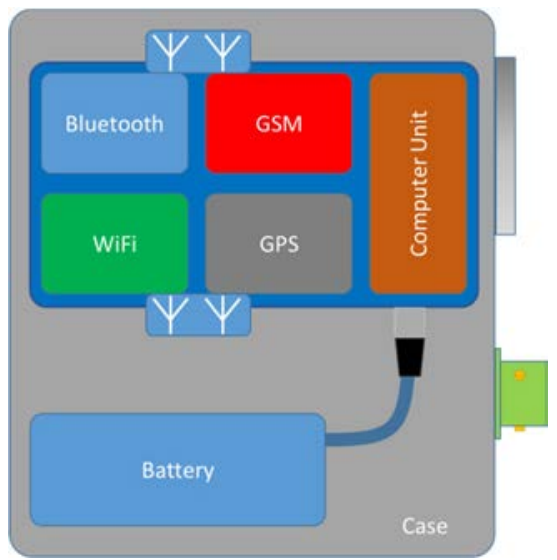

(c)

Figure 2: Bluetooth monitoring system. (a) Exterior view; (b) Interior view; (c) System design. (Source: DLR.)

is added to encapsulate the main sensor module in order to be independent from weather conditions. With built-in rechargeable battery (light blue bow) the system can be run in selfcontained operation. The sensor is deployed in a stationary manner at the roadside.

Due to the MAC's unique character, re-identification of traffic participants can easily be done enabling the collection of spatiotemporal traffic data (e.g. trajectory data of observed vehicles can be subsequently processed to origin destination matrices, route paths and flows, travel times and speed etc.). From the comparison of the derived travel times, the traffic condition on the road can be reconstructed as well (Fig. 3). If the derived travel time is higher than a pre-determined ideal value, congestion or slow-moving traffic can be assumed. In case of free (or nearly free) traffic flow, the travel time is more or less equal to the ideal value [5], [6].

Since the activation of radio frequency interfaces of relevant electronical devices is a necessary condition for a successful detection and since not every traffic object/participant that carries such an appropriate device actually activates those interfaces, just a certain 


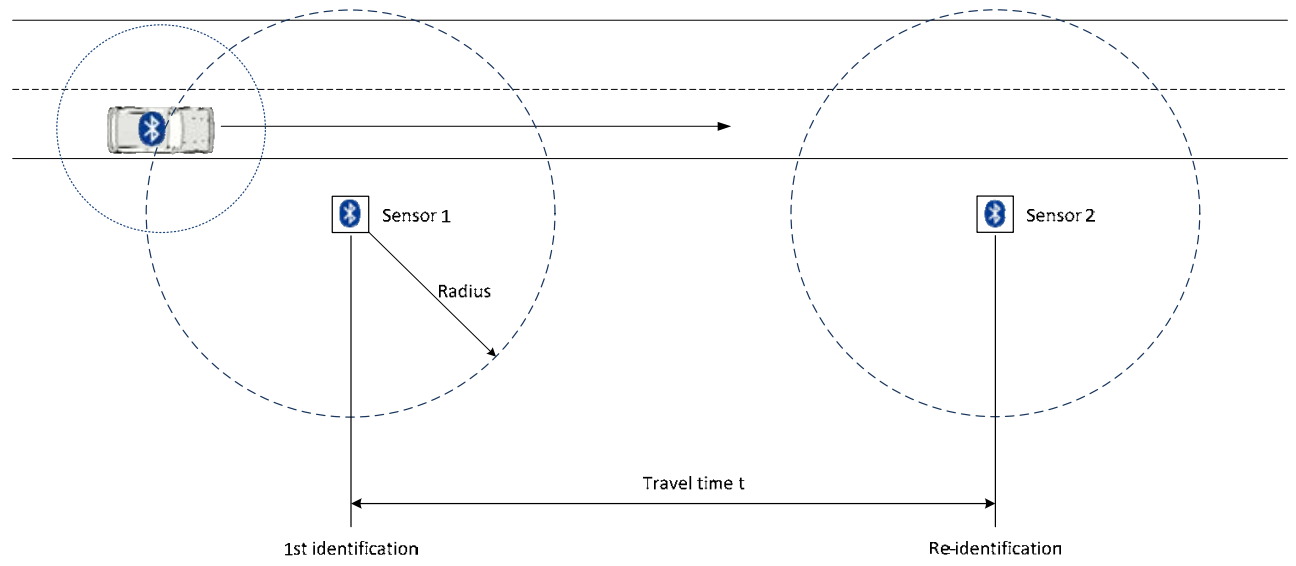

Figure 3: Principle of travel time derivation from re-identification. (Source: DLR.)

amount of the overall traffic volume can be observed using that kind of monitoring system [3], [4]. Related to the use case described within this paper, the detectable portion of traffic volume was $25 \%$ derived from a reference measurement done parallel to the monitoring of arrival traffic flows.

Since all observations are made indirectly while traffic objects pass the monitoring system, the Bluetooth-based monitoring provides an efficient way to collect traffic information in operational traffic management, as well as for long-term traffic and transportation planning in urban areas. Moreover, Bluetooth/WiFi traffic monitoring is a relatively cheap and easy to install solution which allows ad-hoc installation and usage even under extreme weather conditions [7].

\section{CASE STUDY DESIGN AND IMPLEMENTATION}

The monitoring system described in Section 2 was deployed at a worldwide renowned open air festival for the purpose of traffic and person monitoring and thus, to investigate the current traffic situation along the arrival and departure roads as well as the number of people at specific local points on the event area. The case study accompanied the operating business of the event management and provided real-time traffic information for the responsible traffic management staff. The aim of this case study was twofold: on the one hand, we aimed at collecting anonymised data of the current traffic situation in order to examine the impact of the availability of such data on the traffic management processes during large scale events. On the other hand, an overall objective was to demonstrate the current status of research and innovation development in a realistic environment and to investigate questions regarding the benefits, the systems suitability and its potential for improvement as well as the possibilities for future enhancements.

The open air festival, which was chosen as scenario for the use case, is one of the world's biggest music festivals and the biggest open air festival in Germany. The average number of participants is 85,000 people, whereas 50,000 arrive with their own cars (according to the information from the responsible traffic management). The annual festival takes place in the first week of August (4-6 August in 2016). Early arrivals start on the Sunday before the event (31 July in 2016). The main arrival days were 2 and 3 August in 2016. The main departure days were 6 and 7 August. 


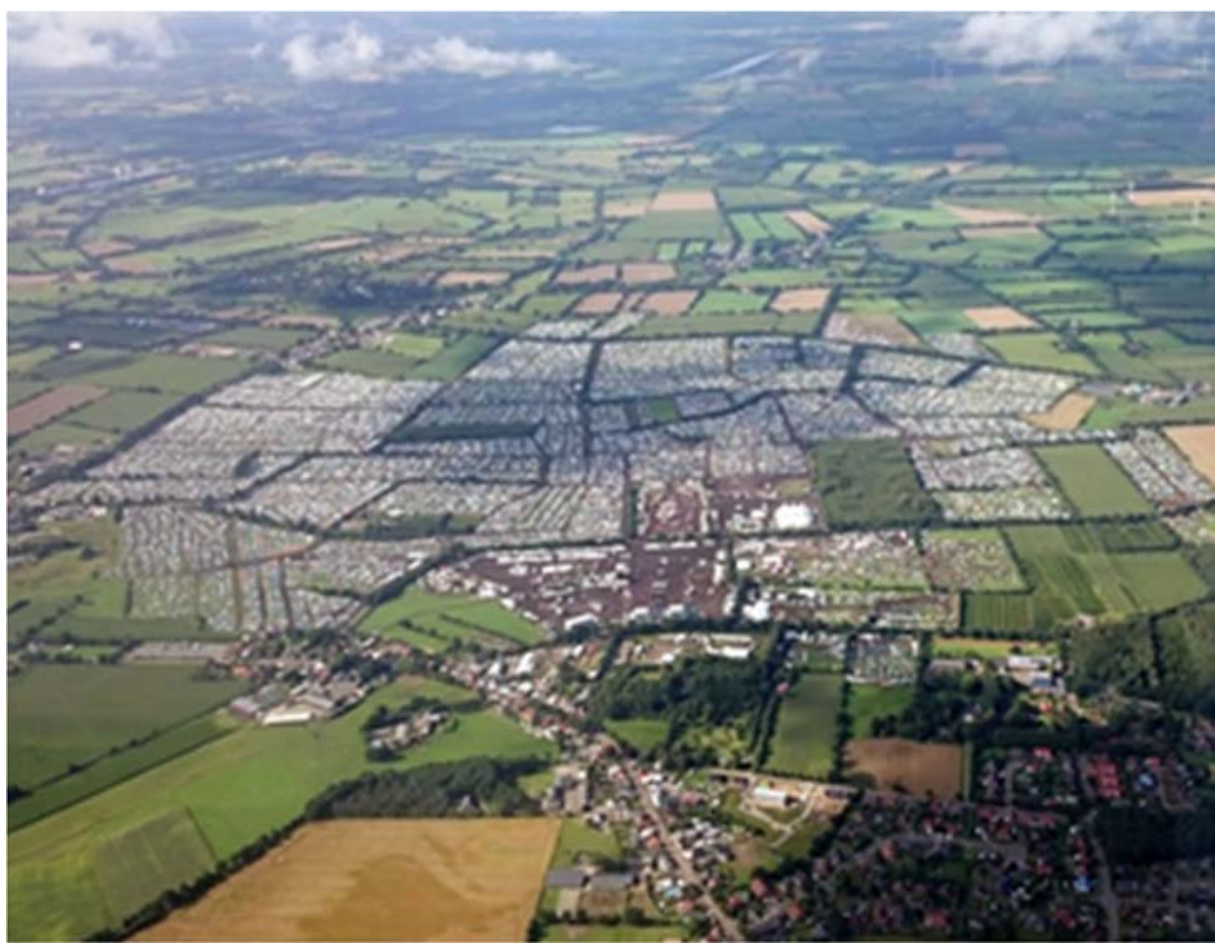

Figure 4: Festival area. (Source: DLR.)

To sum up, the objectives of the use case were as follows:

- Estimation of pedestrian flow at local points on the festival area;

- Derivation of daily courses;

- Derivation of peak loads at the entrances of the festival's infield.

The measurement setup included 12 Bluetooth monitoring systems with ten devices being deployed at the arrival/departure roads to/from the festival area in order to support the local traffic management (Fig. 5, numbers 1-10) and two devices being deployed at the two entrances of the festival's infield, i.e. at the security gates where the festival visitors enter the distinct area in front of the stage (Fig. 5, letters "c" and "d" and Fig. 6). For the detection of local pedestrian densities, the amount of sensors was limited to two devices from the event management side.

Since the festival's infield was not opened before the beginning of the stage events (on 4 August 2016 at 2 pm, Fig. 6), the sensors placed at the entrances of the infield operated only during the actual festival days, i.e. from 4-6 August 2016. Therefore, data from these sensor positions was gained only during this period of time. Moreover, for these two sensors class 2 Bluetooth adapters with a detection range of around 25 meters were used whereas for all the other sensors class 1 adapters were deployed. According to the specifications of the event management, the sensor devices at the entrances to the infield operated only in offline mode. That means that proper functionality could not be checked through remote access but had to be regularly checked via optical appraisal. Therefore, possible system failure were only recognized and solved with time lag (Section 4). 


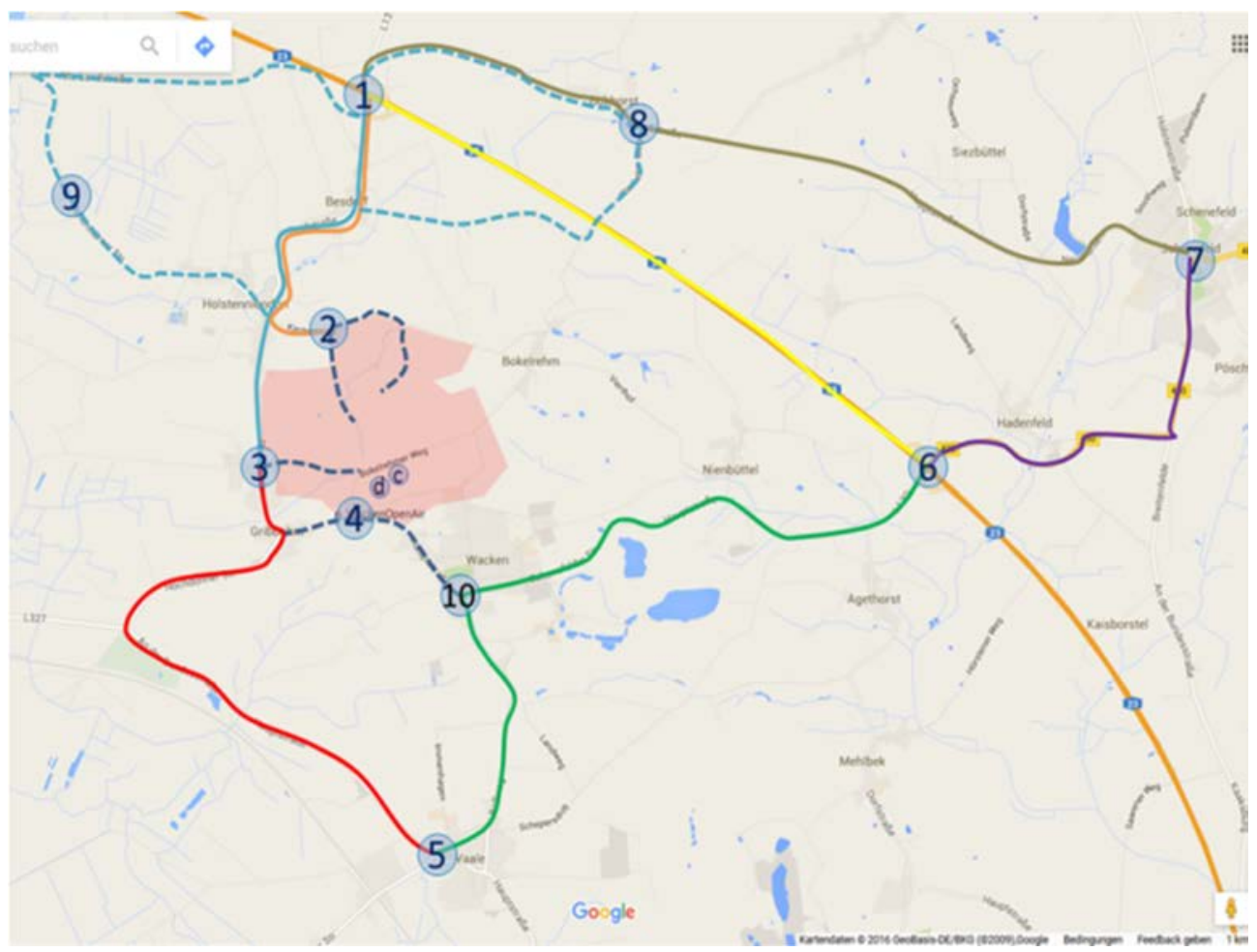

Figure 5: Sensor positions. Numbers 1-10, at the arrival/departure roads; Letters "c" and "d", at the festival's infield for the purpose of pedestrian monitoring. (Source: DLR using Google Maps.)
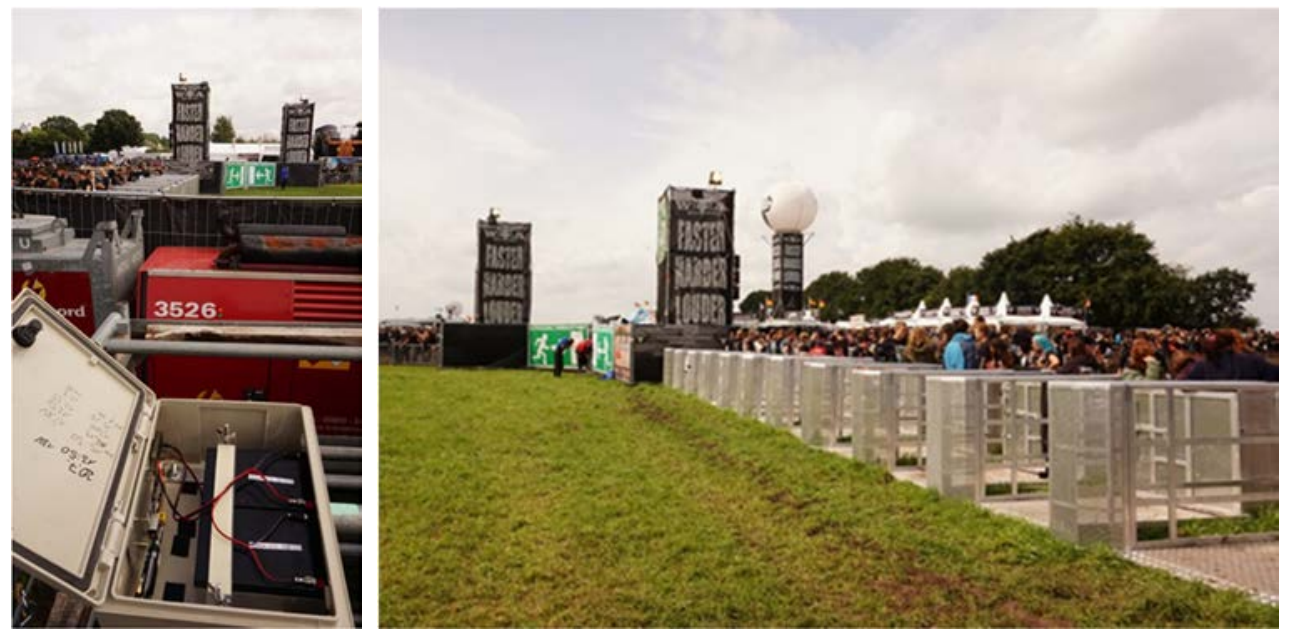

Figure 6: Sensor positions at the entrances of the festival's infield. (Source: DLR.) 


\section{RESULTS}

The daily courses derived from the data collected at the entrances of the infield illustrate the distribution of visitors during the days of the festival in the surrounding of the sensors. Significant peaks can be identified and associated with particular stage events. For example, a notable increase of the amount of visitors was recognized on the first day (4 August) at both entrances between 12 am and $3 \mathrm{pm}$. At that day, the entrance was opened the first time for the visitors at $2 \mathrm{pm}$. Optical reference observations made at the same time confirmed that the visitors started to crowd in front of the entrances already 1 to 2 hours before the actual opening (Fig. 7). With the beginning of the first stage event at $3 \mathrm{pm}$, the number of people flowing into the infield area declined. This observation was made at both entrances in the same manner (Fig. 7, upper and lower diagram for 4 August). From 5 pm onwards an increase of the amount of visitors was recognized once again. Reasons were likely new inflowing visitors or visitors getting out of the infield area. Based on the collected data, an unambiguous answer is not possible since the direction of motion requires a re-identification of the detected device at another sensor and thus, is not derivable from only one sensor. Due to the restrictions made by the event management, such a sensor constellation was not intended to be deployed. However, based on the optical observations made on-site, the second increase can be related to the stage programme since the bands in the time frame between $6 \mathrm{pm}$ and $11.30 \mathrm{pm}$ were major attractions.

On the second day, peak loads occurred in particular in the afternoon from $1 \mathrm{pm}$ at both entrances. One should note that the sensor at entrance ' $d$ ' had a system failure between 4 August from 9 pm until 5 August at 1 pm (Fig. 8). Therefore, the sensor had to be rebooted. Sensor "c" was faced with system failures as well. However, these failures lasted only short periods of time with a maximum of 4 hours (Fig. 7). Since the sensor devices at the entrances of the infield did not run in online mode (which was a pre-condition from the event management side), the proper functioning could be checked only on-site. At the same time, the available DLR staff on-site had to regularly check all the other sensors deployed at the arrival/departure roads around the festival area, and had to exchange batteries if needed. The replacement of batteries was a time consuming task due to the spatial distances between the sensors and the current traffic situation on the roads. Therefore, the recognition of system failures took a lot of time, especially in the infield where the sensors were only accessible on foot.

Nevertheless, the daily course of visitor numbers at the sensor position "c" on 5 August shows for the hours of the night and the next morning (from $12 \mathrm{pm}$ to $11 \mathrm{am}$ ) that less visitors were in the surroundings of the entrance to the infield. Therefore, it can be assumed that the amount of people in the surroundings of sensor ' $d$ ' showed a similar behaviour during system failure. Moreover, the further progression of the amount of detected people indicates that assumption as well. Between $3 \mathrm{pm}$ and $4 \mathrm{pm}$ both sensors detected almost the same amount of visitors, and from $4 \mathrm{pm}$ up to $5 \mathrm{pm}$ the numbers of detected people decreased at both entrances to the same extent.

Between $5 \mathrm{pm}$ and $8 \mathrm{pm}$ on 5 August once again data from only one sensor position ("d") were available. However, in connection with the stage programme a strong correlation between the amount of visitors and acting bands is evident again. Observations made on-site confirm that the clearly identifiable peak between $5 \mathrm{pm}$ and $6 \mathrm{pm}$ on 5 August relates to a famous band that caused a lot of visitors to enter the infield.

Basically, the amount of detected people was permanently on a high level and thus, reflects a high fluctuation in the surroundings of the entrances to the infield. This assumption was likewise supported by the observations made on-site and was possibly caused by the 


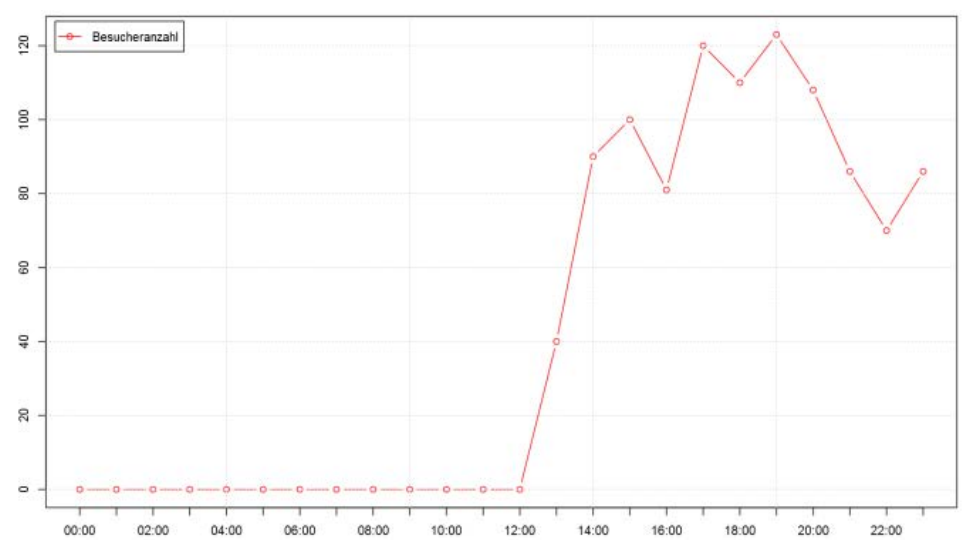

(a)

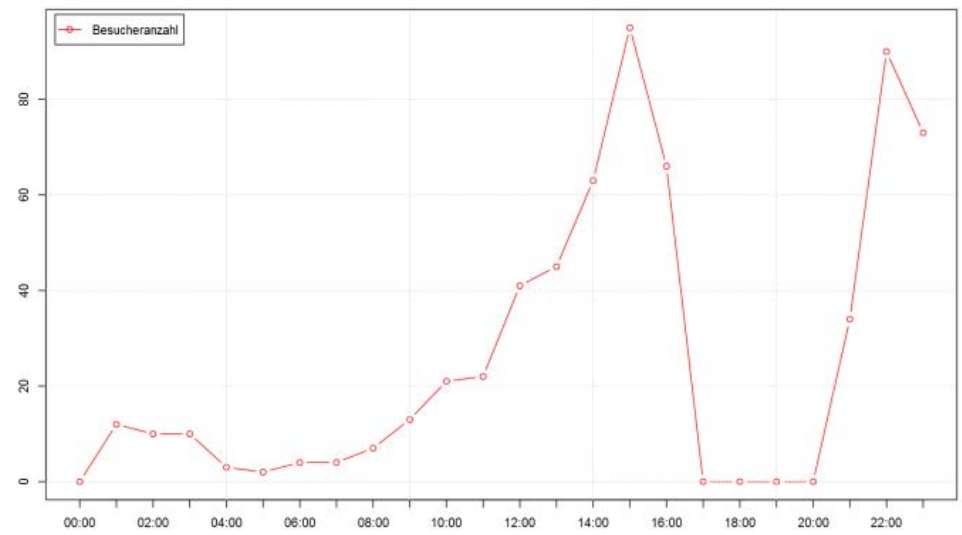

(b)

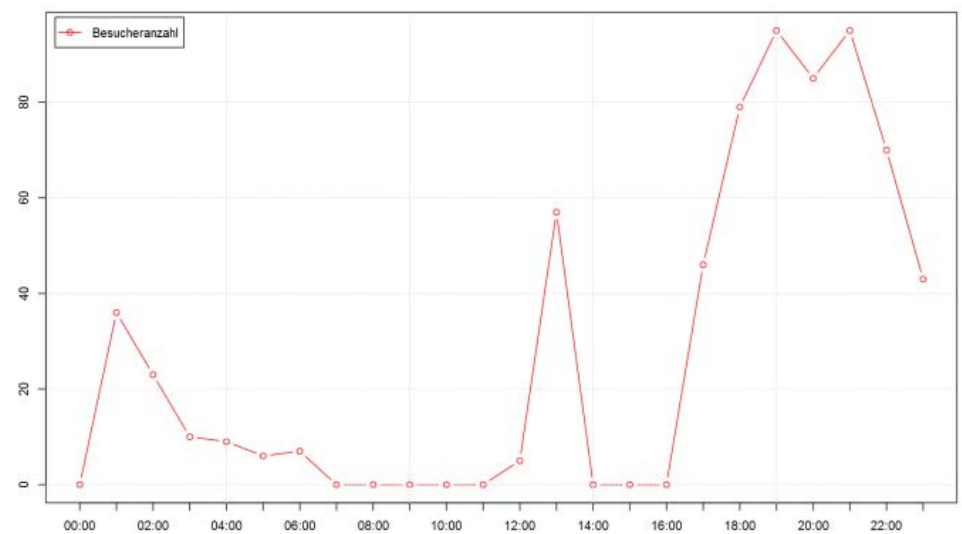

(c)

Figure 7: Daily courses of detected pedestrians at entrance "c" of the infield (vertical scale: detections, horizontal scale: time of the day). (a) 4 August 2016; (b) 5 August 2016; (c) 6 August 2016. 


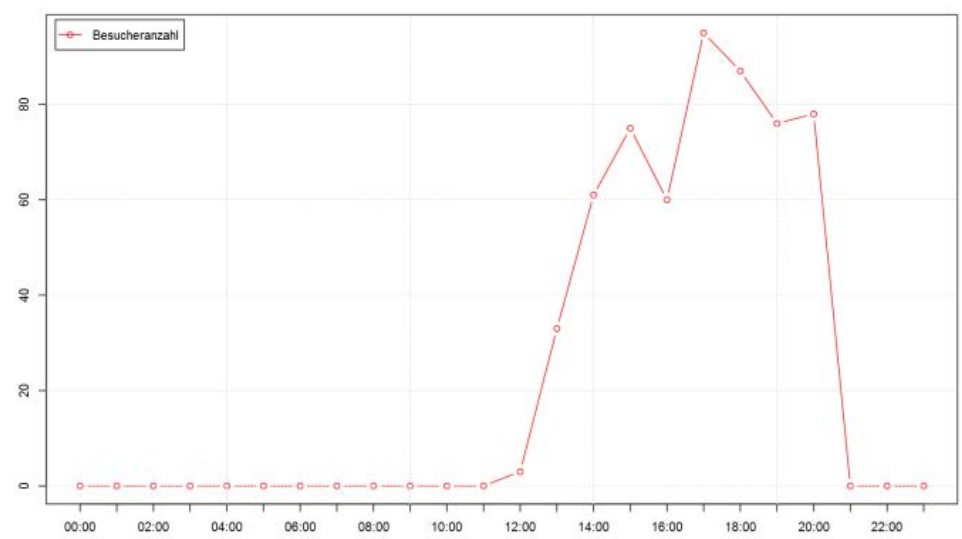

(a)

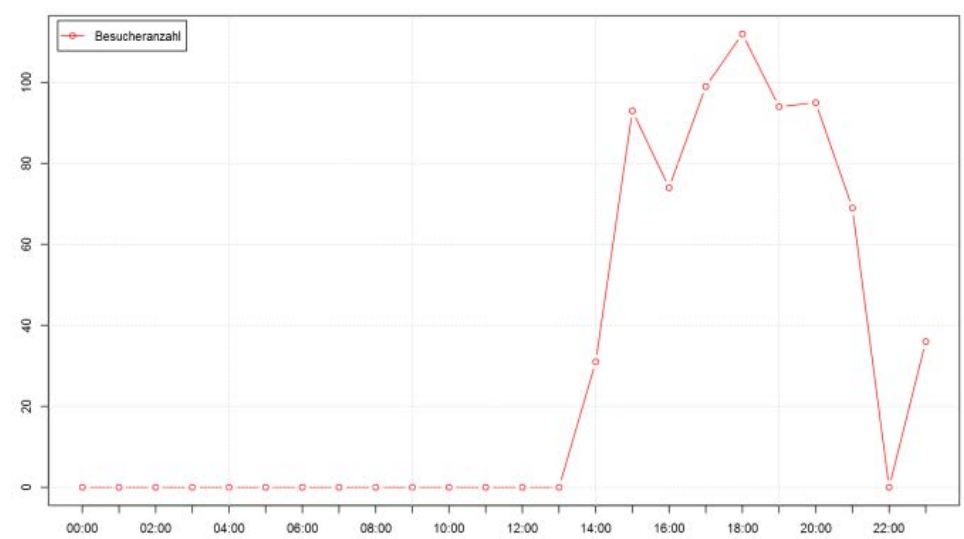

(b)

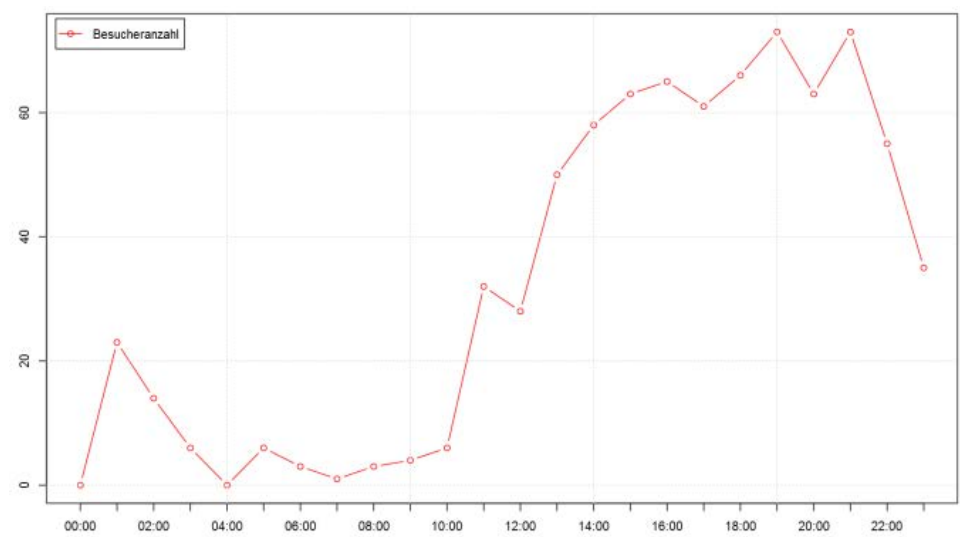

(c)

Figure 8: Daily courses of detected pedestrians at entrance " $d$ " of the infield (vertical axis: detections, horizontal axis: time of the day). (a) 4 August 2016; (b) 5 August 2016; (c) 6 August 2016. 


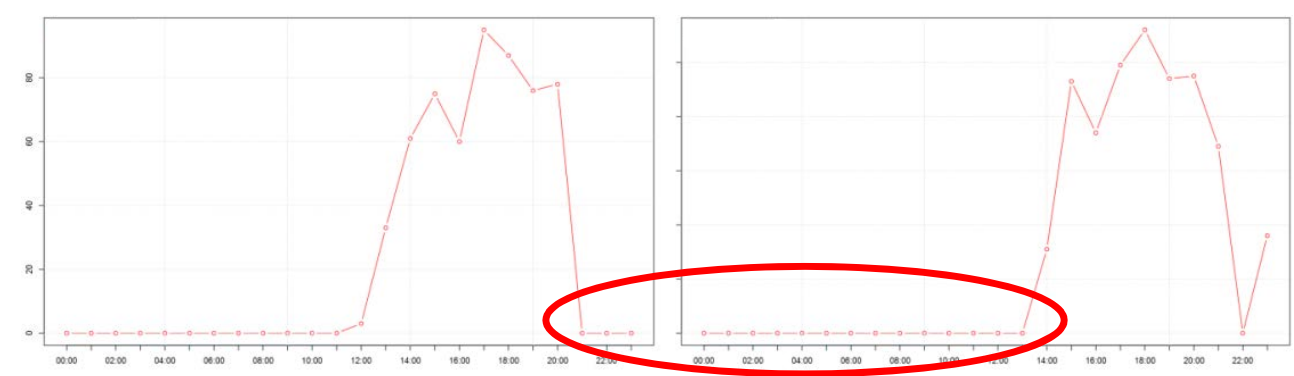

Figure 9: System failures during the operating hours of the infield sensor at entrance ' $d$ ' (vertical axis: detections, horizontal axis: time of the day). Left: 4 August 2016. Right: 5 August 2016.

stage programme that addressed different age groups in constant change between $4 \mathrm{pm}$ and $9 \mathrm{pm}$. Another peak that occurred in the evening around $10 \mathrm{pm}$ (Fig. 7, 5 August at entrance "c") relates once again to a specific band that addresses a wide audience.

On the last day of the festival (6 August) high peak loads were also recognizable in both the afternoon hours and in the evening from $6 \mathrm{pm}$. Here, the optical observations confirmed again that two bands, which were on stage from $7.30 \mathrm{pm}$ respectively from 10.30 $\mathrm{pm}$, attracted a huge amount of people. The constant high level of detections made in particular at entrance " $d$ " during these hours might be an indication for a high fluctuation of visitors between $3 \mathrm{pm}$ and $7 \mathrm{pm}$ once again. Following the observations made in this time, the reason for the fluctuation was due to stylistic changes in the stage programme, i.e. different target groups.

To sum up, the results made by the Bluetooth-based monitoring system depict the current situation on-site in an adequate way. In connection with the observations made by the DLR staff parallel to the automatic measurements, daily courses of the amount of detected people could be derived and interpreted. It turned out that peaks in the daily course relate to specific stage events. However, interpretations regarding the direction of movement of the detected people (i.e. a clear assignment if the detected person entered or left the infield area) as well as a proper data referencing remain open. Thus, the definite percentage of people detected in relation to the overall amount of all available persons in the festival area could not be derived. Based on the random sample done by optical reference observations, a detection rate of $25 \%$ was estimated. It has to be taken into account that this rate is not fully reliable and thus, cannot be transferred directly to other use cases.

\section{CONCLUSION}

With the results of the paper, the authors want to contribute to the research on safety concepts for large scale events. Therefore, we demonstrated that with relatively little effort and low costs an ad-hoc sensor network can be installed and operated, which allocates reliable information on the current situation for the event management and, thus provides an adequate data base for professional responders in order to efficiently initiate and execute evacuation measures in case of potential risks. It turned out that the usage of locally deployed Bluetoothbased sensors allows adequate continuous time measurement, which enables a reliable registration of situation changes in the local concentration of people over the course of the day. The findings are accompanied by referencing results from observations, which were 
made by the staff on-site and which support the results made from the Bluetooth-based monitoring.

Based on the investigations done in an operative environment of a realistic use case, the authors like to provide the following recommendations for further measurements:

- From event management site, privacy concerns regarding the collected data are one the most challenging points when setting up such an experiment. Detailed explanations as well as an approval from a data protection officer are recommended.

- For the derivation of Bluetooth/WiFi-based direction-related motion profiles of detected people, sensor constellations have to be taken into account that allow identification and re-identification of relevant devices. Therefore, at least two sensors in a line must be deployed.

- Operating sensor systems in online mode allow fast reaction in case of system failures.

\section{ACKNOWLEDGEMENT}

We thank ICS Festival Service GmbH for the possibility to connect research with operative management and to allow us to set up our monitoring system at one of their most famous music festivals.

\section{REFERENCES}

[1] Wasson, J.S., Sturdevant, J.R. \& Bullock, D.M., Real-time travel time estimates using media access control address matching. Institute of Transportation Engineers Journal (ITE Journal), 78(6), pp. 20-23, 2008.

[2] Bluetooth Special Interest Group (SIG), Specification of the Bluetooth system, core version 1.2 and higher, 2004, Online. www.bluetooth.com.

[3] Duflot, M., Kwiatkowska, M., Norman, G. \& Parker, D., A formal analysis of Bluetooth device discovery. International Journal on Software Tools for Technology Transfer, 8(6), pp. 621-632, 2006.

[4] Franssen, A., Impact of multiple inquirers on the Bluetooth discovery process - and its application to localization, University of Twente, 2010.

[5] Hoyer, R. \& Leitzke, C., Verfahrenstechnische Bedingungen für die Reisezeitbestimmung mittels Bluetooth-Technologie. Proceedings of the Heureka Conference 2011, Kassel, Germany, 2011.

[6] Haghani, A., Hamedi, M., Farokhi Sadabadi, K., Young, S. \& Tarnoff, P., Freeway travel time ground truth data collection using Bluetooth sensors. Proceedings of the TRB 2010 Annual Meeting, College Park, Maryland, US, 2010.

[7] Gurczik, G., DYNAMIC - Ad-hoc network-wide traffic detection. Presented at EUSTO International Conference 2016, Dresden, Germany, 2016.

[8] Versichele, M., Neutens, T., Delafontaine, M. \& Van de Weghe, N., The use of Bluetooth for analysing spatiotemporal dynamics of human movement at mass events: A case study of the Ghent Festivities. Applied Geography, 32(2), 2012. 